\title{
„EIN MONOLOG DES FÜRSTEN MYSCHKIN ““ (NACH FEDOR DOSTOEVSKIJS ROMAN IDIOT) VON INGEBORG BACHMANN UND SEINE ÜBERTRAGUNGEN INS RUSSISCHE: ASPEKTE DER ÜBERSETZUNGSFORSCHUNG
}

\author{
Andrea Meyer-Fraatz, Jena, Deutschland \\ andrea.meyer-fraatz@uni-jena.de
}

Original scientific paper

DOI: 10.31902/fll.38.2021.2

\begin{abstract}
Der Beitrag untersucht drei Übersetzungen eines Fragments aus Ingeborg Bachmanns Libretto zu Hans Werner Henzes Ballett Der Idiot. Bachmanns Libretto basiert zwar auf dem Roman Dostoevskijs, bringt aber auch zentrale Themen ihrer eigenen Lyrik zum Ausdruck, was sich nicht zuletzt darin zeigt, dass der Text in den Gedichtband Anrufung des Großen Bären integriert wurde. Im Sinne des transferorientierten Ansatzes der Göttinger Schule zur Übersetzungsforschung werden zunächst der Ausgangstext und seine Sinnstrukturen in den Blick genommen, um sodann die Übersetzungen im Hinblick auf signifikante Abweichungen zu untersuchen und diese aus der literatur- und kulturgeschichtlichen sowie gesellschaftspolitischen Situation heraus zu erklären. Es zeigt sich, dass offensichtlich die Tatsache, dass Bachmanns Text auf Dostovskij zurückgeht, eine nicht geringe Rolle für seine Auswahl in den jeweiligen Publikationen gespielt haben dürfte.
\end{abstract}

Schlüsselwörter: Ingeborg Bachmann; Hans Werner Henze; Fedor Dostoevskijs Idiot; Übersetzungsforschung; Elizaveta Mnacakanova; Irina Grickova; Elizaveta Sokolova

I.

Mit Ingeborg Bachmanns „Monolog des Fürsten Myschkin“ liegt ein Text vor, der zunächst davon zeugt, dass das Werk Fedor Dostoevskijs gewissermaßen im deutschen Kulturkreis angekommen ist und man seine Kenntnis voraussetzen kann. Einerseits ist Bachmanns Text Resultat von Übersetzungen Dostoevskijs ins Deutsche und andererseits

\footnotetext{
${ }^{1}$ In Bezug auf den Text Ingeborg Bachmann verwende ich die dort gewählte Dudentranskription, während ich bei Nennungen russischer Namen in Bezug auf Dostoevskijs Roman oder die Übersetzungen von Bachmanns Text ins Russische die wissenschaftliche Transliteration verwende.
} 
wiederum hat er zu Übersetzungen ins Russische geführt. Diese komplexe Situierung eines auf Dostoevskij basierenden Textes zwischen zwei Kulturen soll im Folgenden zum Anlass genommen werden, ihn auf der Grundlage der Übersetzungsforschung zu untersuchen. Dabei spielt nicht zuletzt die Bedeutsamkeit, die das Werk Fedor Dostoevskijs durch seine Rezeption im deutschsprachigen Bereich entwickelt hat, eine wesentliche Rolle.

Vor Friedrich Schleiermachers bahnbrechendem Aufsatz „Die Aufgabe des Übersezers [!]“ (Schleiermacher 1973) hatte man sich nur sporadisch mit dem Problem des Übersetzens beschäftigt. Zwar lassen sich seit der Antike Bemerkungen zum Übersetzen dokumentieren, jedoch hat sich bis in die Zeit der Aufklärung hinein, etwa bei Gottsched, keine wesentlich andere Auffassung vom Übersetzen als bloßem Austausch von Wörtern entwickelt (vgl. Apel 37-38). Mit Übersetzungstheorien vor und nach Schleiermacher setzt sich Friedmar Apels Monographie „Sprachbewegung“ von 1982 auseinander. Nicht zuletzt diese Arbeit hat dazu beigetragen, die Übersetzungsforschung zu einem Teil der Literaturwissenschaft werden $\mathrm{zu}$ lassen. Am Sonderforschungsbereich 309 „Die literarische Übersetzung “ wurde der sogenannte „transferorientierte Ansatz" entwickelt, der als Göttinger Schule der Übersetzungswissenschaft inzwischen in Handbüchern erläutert wird (vgl. Lorenz 568-569, Stolze 155-157). Dieser Ansatz soll Grundlage der folgenden Übersetzungsvergleiche von Ingeborg Bachmanns „Monolog des Fürsten Myschkin“ werden.

Ausgangspunkt für den transferorientierten Ansatz ist die Erkenntnis, dass Übersetzungen notwendigerweise von den Ausgangstexten abweichen und eine Äquivalenz nur selten gegeben ist. Die unterschiedlichen Sprachen, literarischen Traditionen und kulturellen Eigenheiten bedingen geradezu das Abweichen der Übersetzung vom „Original“. Gerade die Differenz zwischen Ausgangsund Zieltext zeigt jedoch, was die Übersetzung als Übersetzung ausmacht: den Transfer eines Textes von einer bestimmten Kultur in eine andere. Mit Augenmerk auf die Differenz lassen sich historisch betrachtet sowohl zeitbedingte Übersetzungsansätze als auch der Umgang mit dem Anderen beschreiben. Anders als beim systemtheoretischen Ansatz, der vor allem in den Arbeiten Itamar Even Zohars und Gideon Tourys aus Israel entwickelt wurde (vgl. Lorenz 565568; Stolze 167-170), geht es nicht darum, die Übersetzung im System der jeweiligen Literatur zu lokalisieren, sondern darum, ihren Beitrag zum Kulturtransfer zu beschreiben. Insbesondere durch die Untersuchung von Mehrfachübersetzungen lassen sich Rückschlüsse auf den Wandel von Übersetzungskonzepten wie auch den Kulturwandel 
ziehen, Übersetzungen aus zweiter Hand verdeutlichen Kulturbeziehungen, auch lassen sich durch gezielte Untersuchungen mehrerer Übersetzungen einzelner ÜbersetzerInnen deren persönliche Profile erstellen, Kanonisierungstendenzen kommen z.B. durch die Analyse von Anthologien zum Vorschein (vgl. Lorenz 569). Der kulturwissenschaftliche Aspekt der Übersetzung als Medium der Fremderfahrung (vgl. Turk, Huntemann/Rühling) ist in jüngerer Zeit um die Aspekte der Genderforschung und der postkolonialen Studien im Zusammenhang mit Übersetzungen erweitert worden (vgl. Stolze 217232).

Eine Übersetzungsanalyse im Sinne des transferorientierten Ansatzes ermittelt also zunächst, inwiefern Ausgangs- und Zieltext voneinander abweichen. Diese Abweichungen können auf unterschiedlichen Ebenen auftreten und unterschiedlich bedingt sein. Zunächst ergeben sich sprachliche Abweichungen allein dadurch, dass sich Sprachstrukturen niemals eins zu eins übertragen lassen. Nicht nur die unterschiedlichen grammatischen Gegebenheiten (auf der Ebene von Morphologie und Syntax) und die verschiedenen semantischen Strukturen (etwa im Hinblick auf Homonymien und Polysemien und damit in Verbindung mit dem Problem der Ambiguität), sondern auch unterschiedliche stilistische bzw. literarische Konventionen allgemein (dazu gehören auch Verssysteme, die Möglichkeit, Reime zu bilden, etc.) spielen eine wesentliche Rolle für die Entstehung von Abweichungen (vgl. Meyer-Fraatz, 25). Auch unterschiedliche kulturelle „Realien“ können dazu führen, dass Übersetzungslösungen gefunden werden müssen, die zu nicht wörtlichen Übersetzungen führen, wobei sich nicht selten in Zielkulturen im Hinblick auf bestimmte Ausgangskulturen Stereotypien im Sinne von Hypercodierungen auch in Übersetzungen zeigen (bei Übersetzungen aus dem Russischen bisweilen auch dann, wenn die entsprechenden Ausgangstexte gar keine Stereotypien enthalten, vgl. Tippner, 49-52; 211-212). Auf jeden Fall erfordert die Übersetzungsanalyse neben der selbstverständlichen Kenntnis der Ausgangsund Zielsprachen auch gründliche Kenntnisse der literarischen und allgemein kulturellen Konventionen beider Kulturen.

Für die praktische Analysearbeit heißt dies zunächst, den literarischen Text in seiner Faktur und seinem Sinngehalt zu erfassen. Dabei kommt unweigerlich die Hermeneutik mit ins Spiel. So wie nach Schleiermacher - modern ausgedrückt - im ersten Schritt des Verstehens, der Divination, zunächst ein intuitives Verständnis entsteht, das im zweiten Schritt, der Konstruktion, durch eine Textanalyse gesichert wird und dann im Schritt der Kombination mit dem intuitiven Vorverständnis abgeglichen werden kann (vgl. Schmid 106-111), muss 
auch bei der Übersetzungsanalyse zunächst der Ausgangstext auf diese Weise erfasst werden. Sodann wird überprüft, inwiefern der Zieltext einen vergleichbaren Sinngehalt vermittelt bzw. wodurch sich Abweichungen erklären lassen.

II.

Das ausgewählte Beispiel problematisiert den Begriff der Übersetzung gleich in mehrfacher Hinsicht. „Ein Monolog des Fürsten Myschkin" von Ingeborg Bachmann (62-67) entstand als Text für Hans Werner Henzes Ballettpantomine „Der Idiot“ nach dem Roman von Fedor Dostoevskij. Dieser Roman lag zur Entstehungszeit des Ausgangstextes bereits in mehreren deutschen Übersetzungen vor. Es ist davon auszugehen, dass Henze den Roman in einer dieser Übersetzungen gelesen hat, um, inspiriert von Motiven des Romans, sein Ballett zu komponieren². Die Übersetzungen von Dostoevskijs Roman leisten dabei einen wesentlichen Beitrag nicht nur für die Vermittlung russischer Literatur, sondern für den Transfer russischer Kultur, indem sie ein bestimmtes Bild von dieser Kultur vermitteln. Durch das Aufgreifen von Motiven des Romans für das Ballett wird deutlich, dass dieser spezifische Teilaspekt russischer Kultur, Dostoevskijs Roman, im deutschsprachigen Bereich gewissermaßen als eingebürgert gelten kann. Es wird zu fragen sein, inwiefern die Übersetzungen dieses Textes ins Russische dessen spezifische Einbettung in den deutschsprachigen Kontext erfassen und wiedergeben können.

Ursprünglich verfasste die russischstämmige Choreographin Tatjana Gsovsky einen Prosamonolog zu diesem Ballett, der bei der

2 Der Katalog der Deutschen Nationalbibliothek verzeichnet zahlreiche Übersetzungem, die, teils in mehreren Auflagen, in der ersten Hälfte des 20. Jahrhunderts erschienen, z.B. von August Scholz (1916), Arthur Luther (1923), Harald von Hoerschelmann (1925), Reinhold von Walter (1925), Gregor Jarcho (1925), Klara Brauner (1928), Fr. Scharfenberg (1929), Rose Herzog (1930), R. Candrela (1951). Spätere Übersetzungen dürften für das Ballettlibretto keine Rolle mehr gespielt haben. Welche Übersetzungen des Romans Henze oder Bachmann gelesen haben, ist letztlich irrelevant für die vorliegende Fragestelllung. Es soll lediglich ein Eindruck von der Beliebtheit Dostoevskijs im deutschsprachigen Bereich und nicht zuletzt seines Romans "Der Idiot" veranschaulicht werden (vgl.

https://portal.dnb.de/opac/showFullRecord?currentResultld=\%22Dostoevskij $\% 22+$ and+\%22Idiot\%22\%26any\&currentPosition=146. 15.10.2021). 
Uraufführung am Berliner Hebbel-Theater 1952 von Klaus Kinski gesprochen wurde (vgl. Bielefeldt 62; Beck 136; Grell 55) ${ }^{3}$. Aufgrund negativer Kritik an der sprachlichen Gestalt dieses Ballettlibrettos bat der Komponist Ingeborg Bachmann, den Monolog neu zu verfassen (Beck 136; Grell 54). Offensichtlich diente Ingeborg Bachmann der ursprüngliche Text von Gsovsky als Vorlage, denn er befindet sich im Nachlass der Dichterin mit deren handschriftlichen Anmerkungen (Beck 138; Grell 55, 68). Gleichwohl überträgt Bachmann den Prosamonolog Gsovskys nicht einfach in lyrische Sprache - Beck (137) spricht von einer „poetische[n] Reflexion über den Gehalt des Dostoewskijschen Romans". Vielmehr nimmt Bachmann - teils im Einklang mit dem Roman, teils aber auch über ihn hinausgehend - wesentliche inhaltliche Veränderungen vor. So streicht sie etwa die Kirchenszene (bei der Fürst Myschkin vergeblich vor dem Traualtar auf Nastassia Filippovna wartet) und fügt stattdessen den Abschnitt ein, der im vorliegenden Beitrag in den Blick genommen wird, nämlich das dritte Bild von insgesamt sieben, die lyrische Reflexion des Fürsten Myschkin über eine Hinrichtung, die er Rogoschin vorträgt und an deren Ende Rogoschin Myschkin zunächst zu töten versucht, dann aber beide ihre Kreuze tauschen. Das Hinrichtungsthema lässt sich nicht nur auf die Episode im Roman, in der Myškin von einer Hinrichtung berichtet (im ersten Teil, Kapitel V), sondern auch auf einen biographischen Einschnitt des empirischen Autors der Romanvorlage, Dostoevskij selbst, beziehen. Die Ablösung des Mordversuchs durch den Kreuztausch steht zudem für die Ambivalenz des Verhältnisses zwischen Rogoschin und Myschkin.

Im Roman spielt sich das im Monolog verdichtete Geschehen etwas anders ab. Dort erfolgt der Kreuztausch vor Rogožins Mordversuch an Myškin, dem letzterer sich durch einen plötzlichen epileptischen Anfall entziehen kann, und über eine Hinrichtung berichtet der Fürst bei der ersten Begegnung mit der Familie Epančin deren Tochter Aglaja. Die Kontamination verschiedener Figuren und Ereignisse lässt sich damit erklären, dass in der lyrischen Transformation das umfangreiche Romangeschehen auf seinen wesentlichen Kern reduziert werden muss. Dabei entstehen zudem semantische Abweichungen zum Ausgangstext des Romans.

\footnotetext{
${ }^{3}$ Ursprünglich war die Rolle Myschkins in Henzes Ballett eine reine Sprechrolle; erst später hat die Choreographin Myschkin in zwei Figuren gespalten, einen Tänzer und einen Sprecher. Gerade die Version, in der Myschkin nicht tanzt, unterstreicht jedoch, wie Michael Mäckelmann in Pipers Enzyklopädie des Musiktheaters feststellt, die Außenseiterposition des Protagonisten (vgl. Grell, 56).
} 
Bereits die Bezeichnung „Monolog“ steht einerseits im Gegensatz zum von Bachtin postulierten Grundprinzip der Dialogizität in Dostoevskijs Romanen ${ }^{4}$, kennzeichnet andererseits jedoch die isolierte Stellung des Fürsten in der ihn umgebenden Gesellschaft. In gewisser Hinsicht treten die Worte Myschkins im Rahmen des Balletts jedoch in Dialog zu den getanzten Figuren (etwa, wenn Myschkin Nastassia Filippovna im vorangehenden Bild beschwört, sich ihm zuzuwenden, sie aber am Ende des Monologs in Rogoschins Arme gleitet). Mit der das Bild einleitenden Regieanweisung wird ein expliziter religiöser Kontext evoziert: Myschkin steigt vor dem Hintergrund einer Ikone eine Leiter hinab, während er seine an Rogoschin gerichteten Worte spricht.

Wie Christian Bielefeldt (86-98) festgestellt hat, eignet sich Ingeborg Bachmann die Romanfigur des Fürsten Myškin insofern an, als sie ihm zentrale Motive ihrer eigenen Lyrik in den Mund legt, insbesondere das Motiv des Verstummens. Damit wird der Text zu einem genuinen Werk Ingeborg Bachmanns, das in einer eher losen intertextuellen Beziehung zu Dostoevskijs Roman steht und in dem selbstreflexiv eigene poetologische und andere Positionen der Dichterin formuliert werden.

Der Monolog ist im Rahmen der Ballettpantomime Henzes ein reiner Sprechtext und wird nicht gesungen. Dies und die Tatsache, dass er in Ingeborg Bachmanns erstem Gedichtband, "Die gestundete Zeit“, abgedruckt wird, ermöglicht es, ihn losgelöst von Henzes Musik zu betrachten, auch wenn, wie in der Literatur bereits festgestellt wurde, im gesamten Monolog immer wieder thematische Bezüge zum Ballett und zu dessen Musik hergestellt werden ${ }^{5}$. Als rein lyrischer Text ist das dritte Bild denn auch fragmentarisch zum ersten Mal für eine Auswahl österreichischer Lyrik ins Russische übersetzt worden, wobei dasselbe Fragment sechs Jahre später in einer Bachmann-Auswahl in neuer Übersetzung erschien. Auch die vollständige Übersetzung erschien in einer Auswahl von Ingeborg Bachmanns Gedichten.

Der aus mehreren, nicht nummerierten Szenenbildern bestehende Monolog beginnt mit Myschkins Identifikation mit einem bestimmten Menschen, der sich im Folgenden als ein zum Tode Verurteilter erweist. Die Identifikation vollzieht sich über die Zeit, den Augenblick der Hinrichtung, den das lyrische Subjekt seinen eigenen erlebten Augenblicken hinzuzählt. Insgesamt in freien Versen verfasst, wird der Monolog

\footnotetext{
${ }^{4}$ Auch im ganz konkreten Sinn kommt Brigitte Schultze (287-295) in ihrer Dissertaion zu dem Ergebnis, dass der Dialog ein zentrales Strukturelement in Dostoevskijs Roman ist.

${ }^{5}$ Bielefeldt (62-74; 79-81) behandelt ausführlich das Verhältnis von Text und Musik in Bachmann/Henzes Balletpantomime.
} 
vor allem durch lexikalische Wiederholungen strukturiert und rhythmisiert. Die Wiederholungen werden in manchen Fällen mit Ambiguitäten verknüpft, und es treten eine Reihe mehr oder weniger hermetischer Metaphern auf. Der Monolog des dritten Bildes (Bachmann, 68-70), das von zwei der drei russischen Übersetzungen als Fragment ausgewählt wurde (das wiederum der Vergleichbarkeit halber der vorliegenden Untersuchung aller drei Übersetzungen zugrundeliegt) schildert den Prozess der Hinrichtung vom letzten Erwachen des Gefangenen über das Henkersmahl und die letzten Vorbereitungen vor dem Gang zum Schafott. Das lyrische Subjekt erkennt Gemeinsamkeiten sowohl mit dem Urteil, also der Instanz, die für die Hinrichtung verantwortlich ist, als auch mit dem Verurteilten, dessen Hinrichtung die dem Tod überlegene Wahrheit des Lebens vorausgeht. Am Ende des Monologs wird ein Kreis zum Anfang hin geschlossen, wenn erneut der Augenblick der Hinrichtung in den Mittelpunkt rückt, der das lyrische Ich verstummen lässt. Der in den Regieanweisungen anschließend angedeutete Mordversuch Rogoschins an Myschkin und der Kreuztausch stehen in Äquivalenzbeziehung zu Myschkins Worten: der Mordversuch zu der wiederholt als Mord bezeichneten Hinrichtung und der Kreuztausch zum christlich konnotierten Henkersmahl - mit den Worten „Fleisch und Wein“ wird implizit auf die Eucharistie verwiesen, und das Wort „Nächstenliebe" ist ebenfalls auf die Gestalt Christi zu beziehen (die der empirische Autor Dostoevskij wiederum mit seiner Romanfigur in Verbindung gebracht hat ${ }^{6}$.

Betrachtet man den „Monolog des Fürsten Myschkin“ als genuines lyrisches Werk Ingeborg Bachmanns, so ergeben sich, wie bereits erwähnt, Bezüge zu ihrem übrigen Werk, dem sie den Monolog gewissermaßen einverleibt hat. Dies wird im Zusammenhang mit den Überstzungsanalysen aufgegriffen.

Vor dem Hintergrund literarischer Traditionen und kulturpolitischer Gegebenheiten des Ausgangstextes einerseits und seiner (Teil-)Übersetzungen andererseits, ergeben sich folgende Fragestellungen, unter denen die Zieltexte untersucht werden sollen: Wie gehen die Übersetzerinnen mit dem freien Vers um, der sich erst in den letzten ca. 20 bis 30 Jahren in der russischen Lyrik in dem Maße etabliert hat, wie er in der Nachkriegszeit bereits in der deutschsprachigen Lyrik üblich war? Wie verhalten sich die Übersetzungen im Zusammenhang mit - auch im Ausgangstext durchaus kritisch gebrochenen - religiösen

\footnotetext{
${ }^{6}$ Raimaund Johann Weinczyk (22) nennt drei Randbemerkungen in Dostoevskijs Entwurf zum Roman, in denen der Autor seine Romanfigur mit Christus vergleicht: (Dostoevskj 246, 249, 253).
} 
Motiven (die in den 70er und 80er Jahren wenngleich nicht grundsätzlich verboten, so doch immer noch problematisch waren)? Welche Abweichungen lassen sich grundsätzlich mit der Entstehung der Übersetzungen unter Zensurbedingungen erklären? Welche Rolle spielt das eigene lyrische Schaffen bei den ersten beiden Übersetzerinnen? Wie wirkt sich die Tatsache aus, dass die letzte Übersetzung von einer Philologin verfasst wurde? $\mathrm{Zu}$ welchen Lösungen kommen die Übersetzerinnen bei Ambiguitäten im Ausgangstext? In welchen Publikationskontexten sind die Übersetzungen erschienen? Inwiefern dienen die Publikationen einer Kanonisierung der übersetzten Autorin?

Die folgenden Ausführungen werden diese Fragestellungen nicht systematisch für die einzelnen Übersetzungen abarbeiten, sondern die Fragestellungen bilden einen eher allgemeinen Rahmen, der für die Einzelanalysen unterschiedlich relevant ist. An einzelnen Beispielen soll dabei demonstriert werden, wodurch Abweichungen zwischen Ausgangs- und Zieltext jeweils begründet sind.

III.

Alle drei Übersetzungen ins Russische sind im Rahmen von repräsentativen Auswahlbänden Ingeborg Bachmanns bzw. der zeitgenössischen österreichischen Lyrik entstanden. Der zeitliche Abstand zwischen den 1975 und 1981 erschienenen Fragmenten sowie der im Jahr 2000 publizierten vollständigen Übersetzung markiert zugleich die politischen Veränderungen, die sich im Laufe der knapp zwei Jahrzehnte, die zwischen ihnen liegen, ereignet haben. Es wird zu fragen sein, inwiefern dies für die Gestalt der Übersetzungen relevant ist.

Die erste, fragmentarische Übertragung erschien 1975 in einer Auswahl zeitgenössischer österreichischer Lyrik des Verlags Progress (Bachman 1975). In eben diesem Jahr emigrierte die Übersetzerin, Elizaveta Mnacakanova, nach Wien (vgl. Gilbert, 43), wo sie bis zu ihrem Tod am 10. September 2019 bis ins hohe Alter lebte. Mnacakanova war Musikwissenschaftlerin und selbst Lyrikerin. Im Wiener Slawistischen Almanach (Mnacakanova 1982) sowie in der Pariser Zeitschrift Apollon77 und anderen im Ausland erscheinenden Publikationsorganen sind ihre russischen Gedichte, die Anfang der siebziger Jahre u.a. auf Initiative Heinrich Bölls außer Landes geschmuggelt worden waren, veröffentlicht; erst 1994 konnten ihre Gedichte auch in Russland erscheinen und wurden 2004 mit dem Andrej-Belyj-Preis ausgezeichnet (Gilbert, 44). In der Emigration begann sie zudem Gedichte in deutscher Sprache zu schreiben. Von Anfang an verwendet Mnacakanova auch im Russischen freie Verse und geht zu einer experimentellen Sonderform 
konkreter Poesie über (vgl. Gilbert, 46-47). Man kann davon ausgehen, dass ihre Übersetzungstätigkeit auch eine Kompensation für nicht mögliche Publikationen des eigenen Schaffens war.

Ihre Übersetzung des Fragments (das als solches nicht gekennzeichnet wird) aus Hans Werner Henzes Ballettpantomime trifft einerseits den Sprachduktus und Rhythmus des Ausgangstextes, verwendet jedoch zahlreiche Hinzufügungen bzw. verändert manche semantischen Zusammenhänge. Im Folgenden sollen einige besonders markante Fälle, bei denen Übersetzungsprobleme vorprogrammiert sind, in den Blick genommen werden.

Bereits der deutsche Ausgangstext enthält die rätselhafte Zeile: „(Kein Gesicht, das abends von innen reift!)“, die zudem einen ambigen Ausdruck enthält. Es bleibt unklar, ob mit „Gesicht“ der Körperteil oder eine Vision gemeint ist, bzw. beide Bedeutungen scheinen in der entsprechenden Textpassage zu oszillieren, denn in den folgenden anderthalb Zeilen scheint sich die Bedeutung des Wortes "Gesicht" auf das Konkretum zu verengen („Bedeckt vom Reif einer Kerkernacht / und frostgrün,“), um dann erneut durch die Worte „weht es dem Morgen entgegen" zur abstrakten Bedeutung zu tendieren und schließlich wiederum „mit dem Gitter über den Augen, die doch dem Himmel / einmal aufgetan waren" den körperlichen Aspekt zu assoziieren (Bachmann, 68). Das im deutschen zweideutige Wort „Gesicht" wird bei Mnacakanova eindeutig zu "lico", was aufgrund der fehlenden bzw. anders gearteten Ambiguität des Wortes im Russischen zumindest im Sinne der Verwendung Bachmanns geschuldet ist ${ }^{7}$. Die unterschiedlichen Sprachstrukturen machen eine Eins-zu-einsÜberstragung in diesem Fall unmöglich. Die spezifische Ambiguität des Wortes „Gesicht“ im Ausgangstext wird im Zieltext kaum kompensiert. Jedoch leuchtet weniger ein, warum das Verb „reifen“ durch „rascvetat “" [aufblühen] und nicht durch „sozrevat" “ [reifen] ersetzt wurde und warum das Verb „wehen“ durch das in der Bewegung stärker auf ein Ziel gerichtete „tjanetsja“ [sich ziehen] wiedergegeben wird. Die sehr freie Wiedergabe der Verse „Die Schritte des Wärters hallen in seiner Brust. / Ein Schlüssel sperrt seinen Seufzern auf“ mit „Povorot ključa v zamke / mgnovenno preryvaet ego dychanie" [Das Umdrehen des Schlüssels im Schloss / unterbricht augenblicklich sein Atmen] erfasst nicht die metaphorische Ausdrucksweise des Ausgangstexts, die eine Ineinssetzung des Gefangenen mit dem Gefängnis impliziert, zumal der hier als zweiter zitierte russische Vers semantisch diametral entgegengesetzt zum Ausgangstext endet: Im Ausgangstext werden die

7 "Lico“ heißt im Russischen „Gesicht" oder „Person“. 
Seufzer durch das Aufsperren ermöglicht, im Zieltext werden sie unterbrochen. Dagegen werden manche rhythmisierenden Strukturelemente wie Wiederholungsstrukturen (insbesondere auch am Ende das anaphorische „und“) in vielen Fällen reproduziert und sogar mit anderen Worten geradezu überkompensiert, wenngleich nicht immer dann, wenn es nicht nur unumgänglich scheint, sondern auch, wenn eine wörtliche Übertragung ohne weiteres realisierbar wäre: So wird das Wort „Gemeinsamkeit" aus der siebten Strophe, das in der achten wiederholt wird, einmal naheliegend mit „obščnost" [Gemeinsamkeit], bei der Wiederholung jedoch mit „svjaz"“ [Verbindung] wiedergegeben. Hingegen wird das erwähnte Verb „tjanetsja“ [sich ziehen], das für das deutsche Verb "wehen“ steht, wiederholt, ebenso das Wort "glaza“ [Augen] als Polyptoton durch „glazami“ [mit den Augen], obwohl das Wort "Augen“ im Ausgangstext nur einmal an der betreffenden Stelle auftaucht.

In der siebten Strophe verwendet Bachmann das Wort „Brett“, auf das der Verurteilte seinen Kopf zu legen hat; dies wird zwar wörtlich mit „doska“ übersetzt, im Russischen kann das Wort „doska“ außer „Brett“ jedoch auch „Ikone" heißen (während im Deutschen eher eine Assoziation zur Theaterbühne entstehen könnte). Inwiefern auf diese Weise eine religiöse Anspielung gleichsam eingeschmuggelt wird, um die durch Fortlassen der Regieanweisungen entfallenden religiösen Motive quasi subversiv zu kompensieren, lässt sich nicht eindeutig sagen.

Aus dem Wort "Mord" für die Hinrichtung (in der Wiederholung zudem in einen syntaktischen Parallelismus eingebettet und damit besonders hervorgehoben) wird "smert“ [Tod]. Hier liegt die Erklärung nahe, dass es angesichts der nur unzureichend aufgearbeiteten stalinistischen Vergangenheit, die in den siebziger Jahren noch sehr viel präsenter war als heute, heikel gewesen wäre, auch in einem ganz anderen Kontext eine Hinrichtung als Mord zu bezeichnen.

Einige bei Bachmann isoliert stehende Ausdrücke erhalten bei Mnacakanova Attribute, z. B. das Wort „medlenno“ [langsam], wenn geschildert wird, wie den Gefangenen der Schlaf verlässt, oder das Wort „poslednego“ [des letzten] vor „pereodevanija“ [sich Umziehen], „,beskonečnoj" [der unendlichen] vor "pravdy“ [der Wahrheit] (sogar wiederholt). Das „weiße Gesicht“ wird umschrieben mit „poblednelo kak bumaga" [erbleichte wie Papier], und die bei Bachmann nicht weiter spezifizierten Gedanken des Gefangenen werden zu „mysli, verno samye nelepye" [Gedanken, sicher die allerdümmsten]. Hier liegt der Gedanke nahe, es handelt sich um eine Identifikation der Worte über den Verurteilten mit ihrem Sprecher. Der Vers „Doch in meiner Sterblichkeit" wird zu "ja tak smerten“ [ich bin so sterblich] - eine 
Konstruktion, die in die Nähe unfreiwilliger Komik rückt, kann doch die Sterblichkeit des Menschen nicht relativiert werden. "Lehren“ bei Bachmann wird zu „lernen“ in der russischen Übersetzung, und dem einfachen "Augenblick, von dem ich spreche" wird noch ein Adressat hinzugefügt.

Manche schlicht falschen Übersetzungslösungen fügen sich gleichwohl in den Rhythmus ein, der dem von Ingeborg Bachmanns Text durchaus nahekommt. Das Fortlassen der Regieanweisungen ist sicher der Zeit geschuldet, in der religiöse Bezüge in literarischen Texten (ungeachtet einer gewissen Öffnung gegenüber der Religion seit der zweiten Hälfte der 60er Jahre) gemieden wurden. Möglicherweise steht mit der Zensur auch in Verbindung, dass, um Assoziationen zum strapazierten Begriff „narod“ [Volk] zu vermeiden, aus „des ganzen Volkes“ "ogromnoj tolpy" [einer riesigen Masse] wird und aus den „verbrecherischen Gerichten“ „verbrecherische Richter" werden (da so nur Einzelfälle, nicht aber ein ganzes System als verbrecherisch bezeichnet werden). Unbeantwortet bleibt die Frage, warum manche poetischen Verfahren, insbesondere bestimmte Metaphern, ignoriert werden, während andere Verfahren erhalten bleiben oder gar überkompensiert werden.

Die zweite, denselben Ausschnitt auswählende Übersetzung wurde angefertigt von Irina Grickova (Bachman 1981). Grickova, Jahrgang 1950, ist selbst Lyrikerin und hat eine Reihe von Gedichten aus dem Deutschen übertragen (http://libgen.ingen.lib.rus.ec/a/293652. 15.10.2021). Die Überschrift verweist auf die Herkunft des Texts aus der Ballettpantomime, wobei wiederum nicht deutlich gemacht wird, ob es sich um den ganzen Text oder nur ein Fragment handelt. Wer als russischer Leser bis dahin allenfalls die erste Übersetzung von Mnacakanova gekannt hat (und nicht den ganzen Ausgangstext), könnte meinen, dies sei der gesamte Text. Die zweimalige Auswahl eben dieses Fragments scheint zu bestätigen, dass in diesem Abschnitt zentrale Motive des Romans wie auch der Ballettpantomime zum Ausdruck kommen. Der auch in der ersten Übersetzung verwendete Untertitel „Myškin raskazyvaet o kazni“ [Myškin erzählt über die/eine Hinrichtung] findet keine Entsprechung im Ausgangstext; auch bleiben wiederum die Regieanweisungen am Anfang und am Ende des Abschnitts unübersetzt, sodass in diesem Fragment lediglich die (vermeintlichen) Worte Myškins über eine Hinrichtung zum Ausdruck kommen. Der Mordversuch Rogoschins/Rogožins bleibt ebenso verschwiegen wie der Tausch der Kreuze beider Figuren. 
Die Übertragung der Verse ist gekennzeichnet von zahlreichen Hinzufügungen und (nicht nur dadurch bedingten) semantischen Abweichungen. So erweist sich bereits im ersten Vers der Ausdruck „sekunda“ statt „Augenblick“ (mig) als zu konkret: Während der Begriff des Augenblicks unbestimmt bleibt in der wenngleich extrem kurzen Zeitspanne, lässt sich eine Sekunde exakt abmessen. Zudem heißt es im Ausgangstext, dass jedem Augenblick ein fremder hinzugezählt werde, während in Grickovas Übersetzung jede Sekunde mit dem Schicksal eines dem lyrischen Subjekt fremden Menschen zusammenwachse (was den Aspekt der Identifikation mit dem Verurteilten unterstreicht). Bei der Übersetzung der bereits besprochenen rätselhaften Zeile Bachmanns „(Kein Gesicht, das abends von innen reift!)“ scheint die Verwendung der Ausdrücke "Vzdor" [Unsinn] und „miraž" [Fata Morgana] für das Wort "Gesicht" in der ersten Zeile der zweiten Strophe und die im Ausgangstext nicht wiederholte Verwendung des Wortes "Gesicht" („lico“) in der zweiten Zeile die Ambiguität des deutschen Wortes kompensieren zu sollen. Allerdings werden in den folgenden Zeilen der Strophe wiederum semantische Veränderungen wirksam, wenn ähnlich wie bei Mnacakanova - das zarte und wenig richtungshafte „weht es dem Morgen entgegen“ durch "ustremlennye k utru“ [dem Morgen zuströmend] ersetzt wird und die vergitterten Augen, die dem Himmel bei Bachmann einfach "aufgetan waren“, in der Übersetzung begierig sind, den Himmel zu sehen („alčuččich videt' nebo"). Weitere semantische Abweichungen ergeben sich durch die Übersetzung des Wortes "Nächstenliebe" (russ.: ljubov' k bližnemu) mit "blagorodstvo" [Edelmut] bzw. des Wortes "Wohltaten" (russ.: blagodejanija) mit "velikodušie" [Großmut]. Insbesondere im ersten Fall mag es damit zu begründen sein, dass der biblische Ausdruck entweder der Übersetzerin nicht geläufig ist oder sie ihn absichtlich mit Rücksicht auf die Zensur vermieden hat; womöglich wurde er auch von der Zensur bemängelt.

Es sind nicht nur semantische Abweichungen in Grickovas Übertragung zu beobachten, sondern auch stilistische. In den zentralen Strophen des Monologs (bzw. dieses Fragments) finden sich im Ausgangstext zum einen immer wieder Rückverweise auf die vorangehenden Strophen in Form von Konjunktionen oder Adverbien. Zum anderen werden bestimmte Wörter wiederholt, die nicht unbedingt zutreffend übersetzt werden, bzw. im Rahmen des lyrischen Texts als Wiederholung auch eine bestimmte Struktur aufbauen. „Nun ist aber eine Gemeinsamkeit zwischen uns / und dem Urteil" wird übersetzt mit "No vse že est' obščnost', rodstvo / meždu nami i prigovorom" [Aber dennoch gibt es eine Gemeinsamkeit, eine Verwandtschaft / zwischen uns und dem Urteil]. Die Hinzufügung von „rodstvo“ [Verwandtschaft] 
als Quasi-Synonym zu „obščnost““ [Gemeinsamkeit] verleiht dem Zieltext wie auch an anderen Stellen einen größeren Wortreichtum. Zudem wird zu Beginn der folgenden Strophe, die im Ausgangstext lautet: „Eine Gemeinsamkeit ist auch zwischen uns / und dem Verurteilten", im Zieltext aus der "Gemeinsamkeit" eine „Einheit“" „No est' i edinstvo mež nami i osuždennym" [Aber es gibt auch eine Einheit zwischen uns und dem Verurteilten]. Zwei Zeilen weiter wird wiederum das zweimal auftretende Wort „Mord“ für die Hinrichtung nur einmal mit „ubijstvo“ [Mord], in der darauffolgenden Zeile jedoch mit "smert “" [Tod] wiedergegeben, und das anaphorische „und“, mit dem in der Schlussstrophe dreimal zwei Verse verbunden werden, fehlt ebenso. Damit wird auf einen wichtigen Aspekt der Rhythmisierung der freien Verse verzichtet. Kompensiert wird dies allerdings durch wiederholten Einsatz von asyndetisch gereihten Synonymen: „ne vzdor, ne miraž" [kein Unsinn, keine Fata Morgana]; „i povlekut, i potaščat ego“ [man zieht ihn sowohl als man ihn auch schleppt]; "obščnost", rodstvo" [Gemeinsamkeit, Verwandtschaft]; „istina, pravda“ [Wahr(haftig)keit, Wahrheit]. Das im Ausgangstext verwendete allgemeine Wort "Brett" für die Richtstätte wird mit dem spezifischen Ausdruck „placha“ wiedergegeben (möglicherweise, um eine Assoziation mit der Ikone im Russischen zu vermeiden). Es fehlt eine Entsprechung der Worte "verbrecherische Gerichte“, was vielleicht auch in diesem Fall eine implizite Assoziation zu juristischen Praktiken der Sowjet-, insbesondere der Stalinzeit vermeiden soll.

Die zahlreichen Hinzufügungen, die den übersetzen Text zum Teil mit Bedeutungen füllen, die dem Ausgangstext nicht eigen sind, könnten darauf zurückzuführen sein, dass eine wortwörtliche Übertragung deutlich kürzere Verse hervorgebracht hätte. Dieses Phänomen lässt sich bei der bisher jüngsten Übertragung, die zwar auch nicht konsequent wörtlich übersetzt, aber deutlich näher am Ausgangstext bleibt, beobachten. Insofern hätte Grickova im Wesentlichen darauf geachtet, durch die Hervorbringung von annähernd gleich langen Versen zumindest dem Rhythmus des Ausgangstextes nahe zu kommen. Durch das Fehlen der Regieanweisungen fehlt jedoch das Motiv des versuchten Mordes Rogožins an Myškin und das des Kreuztausches. Dies entspricht allerdings ganz den Gepflogenheiten des sowjetischen Kulturbetriebs auch noch der frühen 80er Jahre, religiöse Themen zu meiden.

Elizaveta Sokolova ist Literaturwissenschaftlerin am INION [Institut für Wissenschaftliche Information in den Gesellschaftswissenschaften] und Übersetzerin zeitgenössischer deutschsprachiger Literatur (http://inion.ru/ru/about/personalities/sokolova-elizaveta- 
vsevolodovna/. 15.10.2021). Ihre Übersetzung aus dem Jahr 2000 ist in einer zweisprachigen Auswahlausgabe von Gedichten Ingeborg Bachmanns erschienen (Bachman 2000). Mit diesem Text liegt der „Monolog des Fürsten Myschkin“ vollständig übersetzt vor, und religiöse Motive wie der Kreuztausch oder auch Begriffe wie das Wort Nächstenliebe sind im Jahr 2000 prinzipiell kein Hindernis mehr für die Publikation.

Umso auffälliger erscheinen vor diesem Hintergrund Abweichungen semantischer Art. So lässt sich auch bei dieser Übersetzung die Problematik um das im Deutschen zweideutige Wort "Gesicht" beobachten. In der Zeile „(Kein Gesicht, das von innen reift!)“ wird in dieser Version das Wort „Gesicht“ mit „,miraž“ [Fata Morgana] übersetzt und, durch Wiederholung in Gestalt des metaphorischen Synonyms, „poroždenie sumerek večernich“ [Ausgeburt abendlicher Dämmerungen] ergänzt. Das Wort „lico“ [Gesicht] taucht hier überhaupt nicht auf, was die folgenden Zeilen noch rätselhafter macht, als sie es im Ausgangstext bereits sind, denn wie soll man sich eine mit Reif bedeckte Fata Morgana vorstellen? Auch verzichtet Sokolova auf das Dem-Morgen-entgegen-Wehen dieses Gesichts, indem sie das Verb „tjanetsja“ [sich verbreiten] verwendet. „Nächstenliebe“ wird bei ihr, wie schon bei Mnacakanova, zu „miloserdie“ [Barmherzigkeit]. Dazu kommt, dass die Kausalität der Verse „Weil er keine Worte hat, / weil keiner ihn versteht, / bringt man ihm Fleisch und Wein" geradezu ins Gegenteil umgekehrt werden: „Slov u nego uže net, / i nikto ego ne pojmet, / no nesut emu mjaso, vino" [ $\mathrm{lhm}$ fehlen schon die Worte, / und niemand wird ihn verstehen, / aber man man bringt ihm Fleisch, Wein]. „Wohltaten“ in der folgenden Strophe werden zu „ščedrye dary“ [großzügige Gaben], und ebenso wenig wie ihre Vorgängerin kann Sokolova etwas mit dem Wort „Vermessenheit" anfangen.

Wie bei Grickova wird anstatt des Pendants für das Wort „Brett“ das semantisch engere, aber durchaus passende Wort „placha“ [Richtstätte] verwendet. Ähnlich wie bei Grickova fehlen desgleichen die durch Konjunktionen hergestellten Bezüge zwischen den mittleren Strophen, und auch Sokolova erkennt nicht die strukturierende Wiederholung des Wortes „Gemeinsamkeit“, das sie zunächst mit „čtoto obščee" [etwas Gemeinsames] übersetzt, beim zweiten Auftreten aber mit „schodstvo“ [Ähnlichkeit] wiedergibt. In der vorletzten Strophe steht jemand vor dem lyrischen Subjekt, während er im Ausgangstext liegt, wobei die chiastische Konstruktion „Und es liegt einer vor mir, / und ich stehe vor einem“ verloren geht. Die Verse „mit allen Möglichkeiten zu dieser Wahrheit / und mit dem Mut zu ihrem Leben / und zu unserem Tode“ werden schließlich zu „i znaju, kak postič‘ ètu istinu: / 
žit' vašej žizn'ju i / prinjat' našu smert" " [und ich weiß, wie man diese Wahrheit erreichen kann: / euer Leben leben und / unseren Tod annehmen]. Abgesehen von der falschen Wiedergabe des kleingeschriebenen „ihrem“ mit der zweiten Person Plural (im Ausgangstetxt bezieht sich das Possessivpronomen auf das Wort "Wahrheit“ in der vorangehenden Zeile), wird hier eine Erklärung des Erreichens der Wahrheit suggeriert, die so im Ausgangstext nicht formuliert ist. Vielmehr steht das lyrische Subjekt vor dem „einen“ mit den zitierten drei Komponenten, ohne dabei etwas erklären zu wollen. Indem die Übersetzerin in der Schlussstrophe nicht wieder das Wort „mgnovenie“ vom Anfang des Abschnitts aufgreift, kann bei ihrer Übersetzung auch keine Ringkomposition des Monologs in diesem Bild entstehen.

IV.

Welche Schlüsse können aus den Übersetzungsvergleichen gezogen werden? Lassen sich prinzipielle Unterschiede der sowjetischen und der postsowjetischen Übersetzungspraxis erkennen? Wie gehen die Übersetzerinnen mit den im Russischen selbst in den 70er und 80er Jahren sich noch nicht vollständig durchgesetzten freien Versen um? Wie lässt sich die mehrfache Übersetzung gerade dieses Textes erklären?

Die erste Übersetzung platziert eine Auswahl von Gedichten Ingeborg Bachmanns in einer Anthologie zeitgenössischer österreichischer Lyrik mit Autoren wie etwa Hugo Huppert, Erich Fried, Paul Celan. Damit wird deutlich, dass die Auswahl eine dezidiert antifaschistische Tendenz aufweist. In den einleitenden Worten wird sogar ganz Österreich als Opfer des Nationalsozialismus dargestellt (vgl. Vitkovskij, 5). Dass auch im Falle der zweiten Übersetzung neben sehr bekannten Gedichten wie „Die gestundete Zeit" der weniger bekannte "Monolog des Fürsten Myschkin“ bzw. zweimal dasselbe Fragment daraus gewählt wurden, hängt sicher mit der Nähe des Textes zur russischen Literatur zusammen. Dabei ist zu beachten, dass es sich bei Dostoevskij in den 1970er Jahren beinahe noch um einen problematischen Autor handelte, der während der Stalinzeit nicht gedruckt wurde und nicht zum Schulkanon gehörte. Erst in den 1970er Jahren wurde eine Akademieausgabe begonnen, die noch immer nicht sämtliche Texte ungekürzt aufwies, und an Schulen und Universitäten wurden nur wenige ausgewählte Werke behandelt. Beide Übersetzerinnen, deren Texte in der Sowjetzeit veröffentlicht wurden, sind selbst Dichterinnen und haben als solche ein jeweils eigenes Gespür für Poesie entwickelt. Bei Mnacakanova kommt noch eine spezifische Musikalität ihres 
eigenen dichterischen Werks hinzu ${ }^{8}$. Damit hängt möglicherweise zusammen, dass sie manche Passagen, wie soeben exemplarisch dargelegt, auf ihre Weise, in beiden Fällen durch Hinzufügungen verändert haben, um damit zumindest den Sprachduktus Bachmanns und die Verslänge zu erhalten. An politisch empfindlichen Stellen vermeiden beide Übersetzerinnen die wörtliche Wiedergabe von Begriffen wie „Volk" bzw. „verbrecherische Gerichte“. Auf diese Weise wird das thematisierte Todesurteil zum kriminellen Einzelfall und lässt sich nicht mit einer verbrecherischen Gerichtspraxis in Verbindung bringen (bei Bachmann ist eine Assoziation zum Nationalsozialismus anzusetzen). Ebenso werden Begriffe gemieden, die einen eindeutigen Bezug zum Christentum herstellen könnten (und darüber hinaus fehlen die das Bild einleitende und beschließende Regieanweisung mit ebenfalls christlichen Motiven).

Die neuere, vollständige Übersetzung des Monologs wurde von einer Philologin verfasst, die auch andere Lyriker aus dem Deutschen übertragen hat. Weitestgehend fällt diese Übersetzung sehr wörtlich aus, obwohl auch sie, wie es scheint ohne Not, an manchen Stellen abweichend übersetzt. In gewisser Hinsicht wird deutlich, dass diese Übersetzung bewusst auf die vorangehenden Fragmente Bezug nimmt, bis hin zu manchen Missverständnissen. Jedoch sind, abgesehen von der falschen Wiedergabe des Wortes „Nächstenliebe“ durch „miloserdie“ [Barmherzigkeit], sowohl die religiösen Motive erhalten (nicht zuletzt auch durch die Einbeziehung der Regieanweisungen) als auch solche Formulierungen, die noch in den 70er und frühen 80er Jahren politisch riskant waren. Wir lesen bei Sokolova durchaus von „verbrecherischen Gerichten“, wenngleich in derselben Strophe aus „des ganzen Volkes“ „Vsech na svete“ [aller auf der Welt] und damit der Begriff des Volkes vermieden wird. Durch die Verkürzung der meisten Verse und einer weniger präzisen Beachtung bzw. Kompensation poetischer Verfahren (vor allem auch der Verzicht auf die Kreisstruktur durch Verwendung anderer Begriffe als die aus der ersten Strophe wiederholten) entsteht ein Text, der - wie allerdings auch in den ersten beiden Fällen - durchaus eine Ahnung von Ingeborg Bachmanns Original ermöglicht, für den Kenner des Ausgangstexts dennoch unbefriedigend bleibt. Da es sich bei dem Band aus dem Jahr 2000 um eine zweisprachige Ausgabe handelt, ist möglicherweise bewusst auf eine Poetisierung des Zieltextes verzichtet worden, was jedoch nicht erklärt, warum durchaus übersetzbare

${ }^{8}$ Gilbert (46) verweist darauf, dass Mnacakoanova selbst davon ausging, dass ihre eigenen Gedichte besser verständlich seien, wenn der Leser/die Leserin auch musikalische Partituren lesen könne. 
Verfahren, die etwa auf Wiederholungen basieren, in der Übertragung nicht berücksichtigt werden. Was den Umgang mit den freien Versen betrifft, so lässt sich bei den beiden Dichterinnen, Mnacakanova und Grickova, eine Tendenz zu einer eigenständigen Modifikation der Rhythmisierung erkennen, während die Philologin Sokolova zwar bemüht ist, Syntax und Semantik zu erhalten, dafür aber weniger „poetisch“ übersetzt.

Eine Begründung für die Publikation von Gedichten Ingeborg Bachmanns wird in der Anthologie von 1975 gegeben, die, bis auf Bachmann, österreichische Autoren versammelt, welche allesamt Verfolgte des Nationalsozialismus waren. Bei Ingeborg Bachmann ist es die angebliche Sehnsucht nach der Heimat des, wie es dargestellt wird, durch Okkupation und Krieg gelitten habenden Landes. Die in der Familie eines überzeugten Nationalsozialisten aufgewachsene Bachmann arbeitet sich jedoch in ihrer Lyrik an dieser Vergangenheit kritisch ab. Dies wird im Vorwort der sowjetischen Anthologie allerdings nicht erwähnt (vgl. Vitkovskij 17-19).

Gleichwohl scheint die Lyrik Ingeborg Bachmanns vor allem in der Sowjetzeit durch ihre für die damalige literarische Situation in Russland höchst innovative Poetik eine Herausforderung für die Übersetzerinnen gewesen zu sein, die, obwohl selbst innovativ, in den Übersetzungen offenbar nicht über das offiziell Zugelassene hinausgehen konnten. Die Übersetzung der Philologin aus dem Jahr 2000 macht einen eher pragmatischen Eindruck und weist deutlich weniger lyrische Qualitäten auf als diejenigen der Dichterinnen. Gleichwohl macht der Auswahlband mit Gedichten Ingeborg Bachmanns aus dem Jahr 2000 deutlich, dass die östereichische Autorin mittlerweile als zum Kanon gehörig angesehen wird.

Die vorgestellten, hier nur auf einige wenige Beispiele reduzierten Übersetzungsanalysen zeigen, dass sich Abweichungen in Übersetzungen in vielen Fällen gleichsam pragmatisch erklären lassen, sei es aufgrund der Unmöglichkeit, bestimmte Wortspiele oder Ambiguitäten zu reproduzieren, seien es Erfordernisse prosodischer Art. Gerade in Literaturen, die unter Zensurbedingungen entstehen, gehen Abweichungen häufig auch darauf zurück, dass bestimmte Tabus unangetastet bleiben müssen. Manche Abweichungen lassen sich allerdings kaum erklären oder allenfalls dadurch, dass der Ausgangstext mit seinen vagen Formulierungen geradezu dazu einlädt, es bei der Übersetzung nicht immer ganz genau zu nehmen. Die Wahl eines zu übersetzenden Textes scheint sich, wie die Mehrfachübersetzungen des „Monologs des Fürsten Myschkin“ zeigen, nicht zuletzt auch mit der Nähe zur eigenen Kultur, in diesem Fall zu einem der auch international 
bekanntesten russischen Autoren, erklären. Indem durch die Übersetzung eines deutschsprachigen Textes, der sich auf einen Roman Dostoevskijs bezieht (und der in den 1970er und 1980er Jahren in der Sowjetunion nicht zu dessen kanonischen Texten gehörte), wird implizit auch auf die Bedeutung des Autors verwiesen, dessen Geburt sich 2021, zum Zeitpunkt der Publikation dieses Beitrags, zum 200sten Mal jährt.

\section{Literatur}

Apel, Friedmar. Sprachbewegung. Eine historisch-poetologische Untersuchung zum Problem der Übersetzung. Heidelberg, Winter, 1982 (= Beiträge zur neueren Literaturgeschichte, F. 3, Bd. 52)

Bachmann, Ingeborg. „Ein Monolog des Fürsten Myschkin zu der Ballettpantomime ,Der Idiot '“. In: dies. Werke 1. Hg. v. Christine Koschel, Inge von Weidenbaum u. Clemens Münster. München, Zürich, Piper, ${ }^{2} 2010$ [1956] S. 62-79

Bachman, Ingeborg. „Monolog knjazja Myškina iz baletnoj pantomimy ,Idiot'“. In: Iz sovremennoj avstrijskoj poėzii. Moskva, Progress, 1975 , S. 298-300

Bachman, Ingeborg. „Monolog knjazja Myškina iz baletnoj pantomimy ,Idiot'“. In: dies. Izbrannoe. Moskva, Progress, 1981, S. 34-36

Bachman, Ingeborg. „Monolog knjazja Myškina iz baletnoj pantomimy ,Idiot'. In: dies. Voistinu. Stichi. Moskau, Nezavisimaja gazeta, 2000. S. $40-69$, bes. S. $50-53$

Beck, Thomas. Bedingungen librettistischen Schreibens. Die Libretti Ingeborg Bachmanns für Hans Werner Henze. Würzburg, Ergon, 1997 (= Literatura. Wissenschaftliche Beiträge zur Moderne und ihrer Geschichte Bd. 3)

Bielefeldt, Christian. Hans Werner Henze und Ingeborg Bachmann: Die gemeinsamen Werke. Beobachtungen zur Intermedialität von Musik und Dichtung. Bielefeld, transcript, 2003

Dostoevskj, Fedor Michajlovič. Polnoe sobranie sočinenij i pisem. T. 9. Idiot. Leningrad, Nauka, 1974

Even-Zohar, Itamar. "Polysystem Studies". In: Poetics Today, 11, 1990, 1, S. 1-268

Gilbert, Annette. Bewegung im Stillstand. Erkundungen des Skripturalen bei Carlfriedrich Claus, Elizaveta Mnatsakanjan, Valeri Scherstjanoi und Cy Twombly. Bielefeld, transcript 2007 (= Schrift und Bild in Bewegung. Bd. 15)

Grell, Petra. Ingeborg Bachmanns Libretti. Frankfurt am Main etc., Peter Lang, 1995. 
Huntemann, Willi u. Lutz Rühling. „Einleitung: Fremdheit als Problem und Programm". In: Dies. (Hgg.). Fremdheit als Problem und Programm. Die literarische Übersetzung zwischen Tradition und Moderne. Berlin, E. Schmidt, 1997), S. 1-25 (= Göttinger Beiträge zur Übersetzungsforschung Bd. 14

Lorenz, Sabine. „Übersetzungstheorie, Übersetzungswissenschaft, Übersetzungsforschung". In: Arnold, Heinz-Ludwig und Heinrich Detering (Hgg.). Grundzüge der Literaturwissenschaft. München, dtv, 1996, S. 555-569

Meyer-Fraatz, Andrea. Die slavische Moderne und Heinrich von Kleist. Zur zeitbedingten Rezeption eines Unzeitgemäßen in Russland, Polen und Kroatien. Wiesbaden, Harrassowitz, 2002 (= Opera Slavica, N.F. Bd. 39)

Mnacakanova, Elizaveta. Šagi i vzdochi. Četyre knigi stichov. Wien, Institut für Slawistk der Universität Wien, 1982 (= Wiener Slawistischer Almanach. Sonderband 6: Literarische Reihe).

Schleiermacher, Friedrich. „Ueber die verschiedenen Methoden des Uebersezens [!]“. In: Störig, Hans Joachim (Hg.). Das Problem des Übersetzens. Darmstadt, Wissenschaftliche Buchgesellschaft, 1973, S. 38-70 (= Wege der Forschung Bd. VIII).

Schmid, Wolf. „Analysieren oder deuten? Überlegungen zur Kontroverse zwischen Strukturalismus und Hermeneutik am Beispiel von Čechovs ,Nevesta“". In: Die Welt der Slawen. 32, 1987, S. 101-120.

Schultze, Brigitte. Der Dialog in F.M. Dostoevskijs "Idiot". München, Kubon und Sagner, 1974 (= Slavistische Beiträge Bd. 76).

Stolze, Radegundis. Übersetzungstheorien. Eine Einführung. 6., überarbeitete und erweiterte Auflage. Tübingen, Narr, 2018

Tippner, Anja. Alterität, Übersetzung und Kultur. Čechovs Prosa zwischen Rußland und Deutschland. Frankfurt am Main, Peter Lang, 1997 (= Slavische Literaturen. Texte und Abhandlungen Bd. 13).

Toury, Gideon. In Search of a Theory of Translation. Tel Aviv, University, Porter Institute for Poetics and Semiotics, 1980

Turk, Horst. „Alienität und Alterität als Schlüsselbegriffe einer Kultursemantik. In: Jahrbuch für internationale Germanistik. Bd. XXII, 1990, S. 8-31

Vitkovskij, E. „Predislovie“. In: ders. (Hg.) Iz sovremennoj avstrijskoj poézii. Moskva, Progress, 1975, S. 5-19.

Weinczyk, Johann Raimund. Myškin und Christus: ein fiktives Gespräch mit J. Ratzinger auf der Basis von F. M. Dostoevskijs Roman "Idiot". Heidelberg, Winter, 2006 (= Beiträge zur slavischen Philologie Bd. 13) 


\section{“PRINCE MYSHKIN'S MONOLOGUE” (AFTER FYODOR DOSTOEVSKY'S NOVEL "THE IDIOT") BY INGEBORG BACHMANN, AND ITS TRANSLATIONS INTO RUSSIAN: ASPECTS OF TRANSLATION REASEARCH}

The paper analyzes three translations of a fragment of Ingeborg Bachmann's libretto for Hans Werner Henze's ballet, The Idiot. Bachmann's libretto is based on Dostoevsky's novel, but it also expresses crucial themes of her own poetry, which is underlined by the fact that the text was integrated into her book of poems, Invocation of the Great Bear. Following the transfer-oriented approach of the Göttingen School of translation research, first, the semantic structure of the source text is examined, after which, the target texts are analyzed with regard to significant deviations, which are then explained against the backgrounds of literary, cultural and social-political history. The fact that Bachmann's text refers back to Dostoevsky's novel plays a not inconsiderable role in the choice of texts to be translated.

Keywords: Ingeborg Bachmann; Hans Werner Henze; Fyodor Dostoevsky's The Idiot; translation research; Elizaveta Mnacakanova; Irina Grickova; Elizaveta Sokolova. 